\title{
LA ECONOMÍA SOLIDARIA COMO DINAMIZADORA DEL DESARROLLO RURAL Y DE LA CONSTRUCCIÓN DE LA PAZ EN COLOMBIA
}

\section{THE SOLIDARITY ECONOMY AS AN ENHANCER OF RURAL DEVELOPMENT AND PEACE BUILDING IN COLOMBIA}

Dustin Tahisin Gómez Rodríguez Universitaria Agustiniana dustin.gomez@uniagustinaina.edu.co

Ehyder Mario Barbosa Pérez

Uaeos

ehyder.barbosa@orgsolidarias.gov.co

Óscar Misael Merchán Hernández

Uaeos

oscar.merchan@orgsolidarias.gov.co

\section{RESUMEN}

El objetivo general es describir la economía solidaria como posible dinamizadora del desarrollo rural y de la construcción de la paz en Colombia en el periodo 2014-2018. La metodología es de corte cualitativa con el método de revisión documental en los primeros apartados y en la discusión. En los resultados se hace un análisis correlacional entre las variables: Valor Agregado y Número de Organizaciones Solidarias teniendo como derrotero la metodología cuantitativa.

La principal conclusión, es que en los territorios donde se han implementado prácticas basadas en la economía solidaria pareciera que mejoran el peso relativo municipal en el valor agregado departamental.

\section{PALABRAS CLAVE}

Desarrollo rural; economía solidaria; territorio, territorialidad.

\section{Códigos JEL: H11, O1, R11.}

Fecha de recepción: 14/12/2020

\section{ABSTRACT}

The general objective was to describe the solidarity economy as a possible promoter of rural development and peacebuilding in Colombia in the 2014-2018 period. The methodology is qualitative with the document review method in the first sections as well as in the discussion and in the results a correlational analysis is made between the variables: Added Value and Number of Solidarity Organizations taking the quantitative methodology as a course.

The main conclusion is that in the territories where practices based on the solidarity economy have been implemented, it seems that they improve the municipal relative weight in the departmental added value.

\section{KEYWORDS}

Rural Development; solidarity economy; territory; territoriality.

Fecha de aceptación: 25/01/2021 



\section{INTRODUCCIÓN}

Desde la década 1950, en el Estado colombiano se pueden identificar dos corrientes que se visualizan en los planes de desarrollo. Por un lado, la Estructuralista, en cabeza de la Comisión Económica para América Latina y el Caribe - CEPAL - y por otro la Neoliberal desde la perspectiva de los Consensos de Washington, ambas desde el paradigma de la modernidad en términos de Kuhn. La primera denominada Sustitución de las Importaciones, égida bajo el enfoque Estructuralista, buscaba el empoderamiento del sector urbano industrial a través de la productividad de alimentos baratos y de mano obra excedente para la generación de divisas para la importación de maquinaria (Kalmanovitz, 2019; Machado, 2005). El modelo promulgaba el proteccionismo por medio de atender en primera instancia el mercado interno, con lo cual se podría, por lo menos en el discurso, generar crecimiento endógeno para enfrentar la altísima dependencia hacia la tecnológica foránea como a los costos (Vergara, 2011).

"La estructura exportadora colombiana se ha diversificado desde el decenio de 1960. Sin embargo, a pesar de que se ha servido del tipo de cambio para impulsar las exportaciones no tradicionales, las exportaciones con base en la explotación de recursos naturales mantienen una importancia relevante en el comercio exterior" (Rendón, 2007:36).

En el mismo sentido, desde la década de 1990 hasta el presente, con un ligero cambio en el gobierno del Expresidente Ernesto Samper 1994-1998, inicia la segunda corriente o modelo, nombrada Apertura Económica. Que se oficializa con el expresidente Cesar Gaviria, desde el año 1990, al que geopolíticamente se le titula Neoliberalismo (Gómez, 2015). El Neoliberalismo se basa en los postulados de los Consensos de Washington como los de Basilea. Esquematizando, el neoliberalismo tiene cuatro objetivos específicos: la libre movilidad de bienes y capitales, flexibilidad laboral, reducción y limitación del mercado como neutralidad de las políticas macroeconómicas (Rendón, Nieto y Ángel, 2005). 
El desarrollo económico y social en Colombia desde la mitad del siglo XX hasta el presente se ha fundamentado en modelos foráneos: Sustitución de Importaciones y Neoliberalismo .En efecto, así como han dado frutos desde la perspectiva o gremio que se le mire, también ha tenido rotundos fracasos trayendo desajustes sociales y económicos, lo que visibiliza la necesidad de vigorizar y ampliar los derroteros del desarrollo económico y social para así poder generar soluciones y aportes al acervo científico del país (Gómez y Rojas, 2017; Barbosa y Gómez, 2020).

Ahora bien, desde la escuela Neoclásica de la economía el sistema capitalista permite por sus mecanismos intrínsecos que el mercado "autorregule" la conjugación adecuada de trabajo y capital más un componente tecnológico para la generación de riqueza (Berzosa, 1999). Por consiguiente, desde estos postulados, existe una interdependencia entre los diferentes agentes económicos cuya expansión repercute positivamente (Martín, 1983). No obstante, se presentan fallas de mercado, es decir, que el mismo no es capaz por sí solo de regular la distribución, la producción y administración de bienes y servicios. Entre las que se destacan están: la ineficiente asignación de recursos, la inestabilidad de los ciclos económicos, las externalidades negativas y la ineficiencia en la provisión de los bienes públicos las cuales se corrigen generalmente con la intervención del Estado (Stiglitz, 2020; Rubio, Téllez y Gómez, 2019).

El mercado puede ser eficiente pero no necesariamente produce resultados socialmente deseables; por ejemplo, los más pobres pueden ser eficientemente explotados por los más ricos (Stiglitz, 2010). En el ámbito rural, estos fallos llevan a que los productores se enfrenten a mayores niveles de incertidumbre que dificultan la toma de decisiones productivas (Amaya, 2013). De ahí que la presente investigación posibilita otras miradas del desarrollo rural teniendo como pivote la economía solidaria (Vargas y Sánchez, 2020; UAEOS Y Documentación e Información de la Economía Social, Púbica y Cooperativa, 2018).

Precisamente, el presente artículo es derivado del Macroproyecto: Análisis, desarrollo, fomento y gestión del talento humano en el municipio de Aracataca, Magdalena desde la economía solidaria. (2020-2022). Entre la Unidad Administrativa Especial de Organizaciones SolidariasUAEOS, entidad adscrita al Ministerio de Trabajo de Colombia y la Universidad Agustiniana-Uniagustiniana. En virtud que el dialogo entre saberes posibilita un mayor dinamismo para aportar a la solución de las problemáticas complejas de la realidad (Maldonado, 2016; 2007), en este caso específico en la lectura del desarrollo rural como construcción de la paz de Colombia desde la óptica de la Economía Solidaria. Precisamente, por los cambios sociodemográficos, sociopolíticos y socioeconómicos de la sociedad colombiana, urge el análisis interdisciplinario de saberes para 
así poder potenciar el desarrollo rural del país y potencializar el tejido social resquebrajado por 60 años de conflicto armado (Bértola y Ocampo, 2012).

"La historia nos deja la enseñanza del fracaso del crecimiento económico como estrategia principal del desarrollo. Esta estrategia consistía en una forma estandarizada de manipulación social, inventada por los países del norte ajenos a nuestra historia, que desconocía por completo el proceso histórico que nos definió, la estructura institucional y las especificidades de la cultura nacional. Esta ha sido la senda que nos ha llevado a "un desarrollo del subdesarrollo". Por ello, se debe rectificar la senda y optar por un modelo de desarrollo autónomo, fundamento en los principios del desarrollo humano como fin último, y en el sector rural como el espacio imprescindible para lograrlo. Si aceptamos el reto habrá razones para la esperanza" (Vergara, 2011:64).

Para terminar, el presente escrito está dividido en varios momentos. El primero ya expuesto que es la introducción, prosigue con el marco teórico o el sustento argumentativo para interpretar cómo analizar el documento donde se identifica y se toma postura de lo que entiende los autores sobre economía solidaria, territorio, valor agregado y desarrollo rural. Continúa con el tercer espacio en cual se efectúa un análisis correlacional entre diferentes variables (Valor Agregado y Número de Organizaciones de Economía Solidaria) en el periodo 2014-2018. Prosiguiendo con la discusión de los resultados frente a las categorías planteadas terminando con unas breves conclusiones y la referencia utilizada.

\section{MARCO TEÓRICO}

Después de 60 años de conflicto interno armado entre actores legales e ilegales en el territorio colombiano por fin se puede observar en el presente la posibilidad de un nuevo país por medio de la construcción de la paz. Al ser la paz un ejercicio multidimensional, necesita de marcos teóricos que entiendan cómo generen soluciones frente a las problemáticas de la ruralidad, ya que en un mundo que tiende a ser globalizado los cambios tecnológicos relativizan las actividades no competitivas según el discurso neoliberal, así como la precaria distribución del ingreso (Stiglitz, 2012; Losada, Valencia y Gómez, 2018), los limitados procesos educativos (Organización de las Naciones Unidas para la Educación, la Ciencia y la Cultura, 2010) han contribuido a que la sociedad colombiana exhiba unos índices socioeconómicos preocupantes ${ }^{1}$ con asimetrías contundentes entre

1 Para el año 2018 la Pobreza Monetaria en Colombia fue del 27\% de la población. El $7.2 \%$ de la población vive en Pobreza Extrema (DANE ,2018). De igual forma, el país posee la 
lo urbano y rural (Molina, Aranda y Lesmes, 2018; Useche, 2008; Pérez y Pérez, 2002). El presente marco teórico tiene como objetivo describir las categorías utilizadas y las líneas argumentativas con las cuales se sostiene el artículo teniendo como eje lo antes mencionado. Por consiguiente, se explica qué se comprende en cada una de ellas, así como los principales autores de las categorías economía social, desarrollo rural, territorio y valor agregado. A continuación, se desarrolla las líneas argumentativas de la economía solidaria:

\subsection{ECONOMÍA SOLIDARIA}

El concepto de economía solidaria (ES) está constituido por experiencias que tienen como base la horizontalidad entre sus integrantes basados en principios de solidaridad y apoyo mutuo (Osorio, 2017). La ES busca fortalecerse por medio de la creación de redes de cooperación constituyendo de esta manera una posibilidad real de construcción de relaciones equitativas y justas entre sus miembros y pone en el centro al ser humano y su entorno medio ambiental. La ES proyecta el desarrollo como un proceso donde los aportantes de factores los ejecute con independencia en contextos asociativos empresariales. De igual forma, contempla un proyecto de mercado democrático y solidario con el fin de modificar la forma de aplicación del desarrollo con una concepción diferente del mismo (Barbosa y Gómez, 2021; Barbosa, Vargas y Gómez, 2020; Caillé, 2009; Álvarez y López, 2018).

En la academia, la ES comienza a ser reconocida como una modalidad de organización colectiva con características distintivas. Amaro (2009) indica que la ES se caracteriza por tres aspectos: 1. Policéntrico: surge de manera autónoma en los territorios compartiendo líneas de base; 2. Polisémico: Porque adquiere diferentes significados dependiendo del entorno en el que se aplique, y 3. Poli expresión: relacionada con el tipo de prácticas. De igual forma, el autor plantea tres tipos de enfoques de la Economía Solidaria estudiadas en la academia, a saber: 1. La versión francófona que propone la idea de una economía plural que compendia Estado, mercado y sociedad civil. Esta versión además es la más desarrollada teóricamente. 2. La versión latinoamericana, más popular y comunitaria, que valora también el carácter de proyecto cultural y ambiental, y 3. La propuesta de Macarronesia (zona compuesta por cinco archipiélagos del Atlántico Norte: Azores, Canarias, Cabo Verde, Madeira e Islas Salvajes) donde la transformación integral y una visión ecocéntrica constituyen sus puntos más importantes. (Fernández Gómez y Parodi, 2020).

mayor concentración de tierras del continente. El Coeficiente de Gini se ha situado entre 0.801 y 0.809 (Oxfam, 2017). 
La ES tiene como antecedente un concepto más antiguo denominado economía social. La Economía Social está relacionada con experiencias formales de mutualismo y cooperativismo. Existe un debate académico que busca concatenar los dos conceptos en uno solo denominado Economía Social (Estivill, 2012; Laville, 2004) pretendiendo dejar atrás una división existente entre los dos aspectos donde se le critica a la ES la perdida de orientación política transformadora y en la que la economía solidaria sí es vista como una alternativa real al capitalismo. "La Economía Solidaria no es una dimensión económica limitada; por el contrario, es una realidad que abarca la unidad del ser, la integralidad del ser, que considera hacer, sentir y pensar" (Vásquez y Jiménez, 2012, p. 21).

Así, la ES se relaciona con varias corrientes teóricas, contextos socioeconómicos y corporativos y prácticas empresariales y asociativas. Al respecto, Laville $(2004 ; 2006 ; 2007)$ indica que la ES "no se limita a un sinnúmero de actividades económicas con finalidad social, sino que se origina en un movimiento ideológico de la economía y la política. En concordancia con estos autores, emerge como una manera de democratizar la economía a partir de responsabilidades cívicas" (Pérez y Uribe, 2016: 538-539).

A partir de lo anterior se puede decir que existen dos enfoques fundamentales en el contexto de la ES: 1. La orientación europea con importantes relaciones en Quebec(Canadá)y, 2. el enfoque latinoamericano, en especial Chile, Argentina y Brasil (Chaves, 2012; 2006). Aunado a lo anteriormente señalado, Razeto (1992) plantea la necesidad de un nuevo modelo de empresa de trabajadores, de asociación Inter cooperativa con nuevos modos de integración para asegurar una transformación del cooperativismo con economías alternativas y solidarias que se erijan como una racionalidad económica diferenciada. La autogestión es el fundamento de esta perspectiva de integración, el autor menciona en especial circuitos económicos solidarios como modelo de integración solidaria.

En la misma línea, Guerra (2014) plantea que el objeto del cooperativismo social asociado es generar desarrollo local y es justo en este aspecto donde este concepto teórico - desarrollo local - juega un papel importante en la investigación. De acuerdo con los conceptos enunciados, el desarrollo local y la economía solidaria pueden llegar a jugar un papel muy importante es la adopción de dinámicas económicas que permitan la asociatividad, solidaridad y unión de un grupo sensible a los vaivenes socioeconómicos de un país en tránsito hacia una etapa de posconflicto como lo es Colombia. Un autor destacado en el ámbito latinoamericano es (Coraggio, 2011). Su trabajo trata sobre la teoría de la Economía Social y Solidaria. El autor plantea que la economía está constituida por la producción, distribución, circulación y consumo con base en principios, instituciones y prácticas. 
Establece que la Economía está en estrecha relación con la cultura y la política, lo que implica que los principios de la organización económica son de orden social y no económico

Existe consenso teórico (Guerra, 2014; Razeto, 1992; Coraggio, 2011) en el planteamiento de la integración de los eslabones de la Economía a partir de principios solidarios como cambio paradigmático de la forma de hacer y pensar la economía. En este desarrollo teórico, la noción de integración económica remite al territorio, a la comunidad y al relacionamiento entre emprendimientos solidarios con el propósito de potencializar las capacidades locales y así satisfacer las necesidades logrando cambios paradigmáticos en la forma de producir y definir las preferencias de consumo (Álvarez, 2016).

\subsection{ECONOMÍA SOLIDARIA EN COLOMBIA}

La Unidad Administrativa Especial de Organizaciones Solidarias-UAEOS es una entidad pública del orden nacional del Estado de Colombia adscrita al Ministerio de Trabajo. Nace en el año 2011 con el Decreto 4122 y su institucionalidad se basa en la Ley 134 de 1931 sobre las sociedades cooperativas. De igual manera, se nutre con la Ley 24 de 1981 con la cual se crea la institución antecesora de la UAEOS, denominada DANCOOP. Asimismo, con la Ley 79 con la que se actualiza la legislación de las cooperativas. Continúa con la Ley 454 de 1998 que regula el marco conceptual de la economía solidaria (Barbosa, Insuasti y Gómez,2020). Precisamente, el artículo segundo de la norma define la economía solidaria como un:

"sistema socioeconómico, cultural y ambiental conformado por el conjunto de fuerzas sociales organizadas en formas asociativas identificadas por prácticas autogestionarias solidarias, democráticas y humanistas, sin ánimo de lucro para el desarrollo integral del ser humano como sujeto, actor y fin de la economía" (Artículo 2, Ley 454 de 1998).

Desde el punto de vista organizacional, la misión de la UAEOS es promover, fortalecer y desarrollar socio-empresarialmente las organizaciones solidarias para generar desarrollo en los sectores y regiones del país con una institucionalidad del sector fortalecida y transversal. Busca el posicionamiento del sector solidario en la contribución de logros de paz y equidad en Colombia. En el marco de las competencias legales asignadas debe diseñar, adoptar, dirigir, coordinary ejecutar los programas y proyectos para la promoción, planeación, protección, fortalecimiento y desarrollo de las organizaciones solidarias en Colombia (Unidad Administrativa Especial de Organizaciones Solidarias, 2018). 


\subsection{TERRITORIO}

Teniendo en cuenta que la categoría territorio representa un aspecto relevante en la descripción que se desarrolla a continuación. Se presenta los principales aspectos teóricos en los que el territorio se visibiliza como factor primordial para el ejercicio investigativo. Por ejemplo, Medeiros (2009) señala que por territorio se entiende "un juego político, un lugar de poder. Definir sus límites, recortarlo, es sinónimo de dominación, de control" (p. 217). De igual forma, Gottmann (1973) establece que pueden representarse de diferentes maneras las relaciones que tienen las personas con su territorio, por ejemplo, en ámbitos como los geográficos, los políticos y los económicos. Sin embargo, advierte que esta es una visión netamente occidental y no debe ser entendida como totalizadora.

Según lo expuesto por Cruz (2007), se puede afirmar que la construcción del territorio pasa por lo social como base para el encuentro de fuerzas políticas, económicas, territoriales y culturales que difieren unas de otras generando entre sí conflictos continuos. Así mismo, (Echeverri 2004; Espinosa ,2009) sostienen que, aunque los territorios pueden delimitarse y demarcarse, estos se definen no tanto por sus fronteras sino por los vínculos de los grupos humanos a un paisaje y a una historia, estableciendo así lazos de unión afectivos y espirituales significativos. Groso modo la categoría territorio es el espacio geográfico limitado por el poder ejecutivo que ocupan un conglomerados de grupos sociales imprimiendo sus subjetividades como intersubjetividades.

\subsection{DESARROLLO RURAL}

El desarrollo rural trata de dar respuesta o concibe solucionar tres puntos neurálgicos con los cuales se diferencia de las demás formas de entender el desarrollo económico como lo son, entre otros, el endógeno, el local, el posdesarrollo, el Biodesarrollo. El primero es erradicar la pobreza extrema del campo por medio de la formación y el bienestar de su población para reducir la inmigración y marginación en las megaciudades. En segundo lugar, incentivar la producción agrícola sostenible, es decir ampliar la cobertura hacia el acceso de los alimentos y por último preservar el capital natural y los servicios ecosistémicos (Ceña ,1993; Trivelli, Escobal y Revesz, 2009).

De igual modo, es necesario ver lo rural más allá de lo agropecuario, por ejemplo, como un entramado donde se entrecruzan lo territorial, el territorio y los ámbitos multisectoriales. Acotando que es necesario superar la idea de que lo rural es lo atrasado y lo urbano es lo civilizado, sino más bien que lo rural esta interrelacionado con lo urbano, para así lograr equidad social (Cárdenas y Vallejo, 2016). En virtud que el desarrollo rural promueve "la equidad territorial, de género y social, en el acceso a bienes, servicios y demás beneficios del 
desarrollo. La equidad es una meta que, en los albores del siglo XXI, está aún muy distante, ya que los procesos de concentración de la riqueza y de los medios de producción siguen siendo factores que prevalecen" (Pérez, 1988: 10). Con el mismo propósito, el desarrollo rural "va más allá de la agricultura, para incluir un sin número de actividades que se desarrollan en las zonas rurales. Así mismo, la actividad agrícola también debe entenderse hoy como multifuncional, dejando de limitarla puramente a lo productivo" (Restrepo y Bernal, 2014:151). Sin olvidar que el desarrollo rural es el diálogo entre varias disciplinas para entender y solucionar las problemáticas rurales desde esta conceptualización. Por eso al efectuar desarrollo rural se necesita no solo de la participación de sus actores sociales y dinamizadores, sino también la conversación multidisciplinaria entre la economía, antropología, sociología, geografía, ecología, política, economía ecológica, dependiendo desde qué marco comprenda el medio ambiente (Manzanal, 2006; Sánchez y Herrera, 2017).

\subsection{VALOR AGREGADO}

La Ley 136 de 1994 que luego fue modificada por la Ley 617 de 2000 determina la forma como se debe categorizar a los municipios en Colombia. En efecto, se basa en criterios poblacionales que relaciona los ingresos libres de destinación. Precisamente, la Ley 1551 de 2012 que la construyó el DANE, calcula el Índice de Importancia Económica Municipal. Que, a su vez, es el peso del Valor Agregado de un municipio dentro del territorio del departamento ponderado por el total nacional. Asimismo, la Ley antes mencionada ejemplifica que se debía categorizar a los municipios a través del indicador y sus resultados fueron la categorización de los territorios cuyo criterio principal es el Valor Agregado Total Municipal (Delgado y Pardo,2014; Laverde et al., 2020).

\section{METODOLOGÍA}

El documento utiliza dos metodologías. Por un lado, tiene una metodología cualitativa con método de revisión documental por matrices (Gómez, Aldana y Rodríguez, 2021; Gómez 2017a; 2017b; 2016) en los primeros apartados del escrito con lo cual se busca identificar las divergencias y las convergencias de los autores desde la línea argumentativa expuesta como el método. En segundo lugar, la metodología es de corte cuantitativo y el método es correlación entre variables: Valor Agregado y Numero de Organizaciones Solidarias. Los datos fueron tomados de la Unidad Administrativa Especial de Organizaciones Solidarias-UAEOS, del Departamento Administrativo Nacional de Estadística- DANE, del Departamento Nacional de Planeación - DNP y el Registro Único Empresarial y Social-RUES-. La ventana de observación fue desde el año 2014 al 2018 (Hernández et al., 2006). 


\section{RESULTADOS}

Existen otros mecanismos para disminuir las fallas del mercado distinto a la intervención estatal que permiten lograr un mayor desarrollo rural y, además, más sostenible en el territorio Uno de estos mecanismos se relaciona con la gestión de la ES en el ámbito rural. En este sentido, y en forma general la ley 454 de 1998 señala, por un lado, dentro de sus principios la propiedad asociativa y solidaria sobre los medios de producción y la participación económica de los asociados en justicia y equidad; y por el otro, en cuanto a sus fines garantizar a sus miembros la participación y acceso a la formación, el trabajo, la propiedad, la información, la gestión y distribución equitativa de beneficios sin discriminación alguna. Las figuras jurídicas que adopta la ES (cooperativas, asociaciones, etc.), tanto a nivel general como en el contexto rural, cumplen el papel de regulación del sistema y contribuyen al ajuste económico estructural del sector agrario como el empoderamiento del territorio y la territorialidad. De acuerdo con lo anterior, las organizaciones solidarias internalizan los costos sociales y producen externalidades positivas, en cuyo caso disminuyen las fallas del mercado enunciadas anteriormente (Osorio, 2017). Es así como estas organizaciones aportan al aumento de los niveles de empleo y el desarrollo rural (Chávez, 2012).

De esta manera se observa que la asociatividad, como eje fundamental de la ES, potencializa la capacidad de los productores para acceder tanto a recursos como a mercados por medio de la integración horizontal y vertical, lo que les permite aprovechar eficientemente a las economías de escala recibiendo los beneficios (disminución de costos de producción, precios, poder de negociación) que ello conlleva (DNP, 2018). Además de los beneficios económicos descritos anteriormente, también están los que se relacionan con temas sociales inherentes al modelo solidario que contribuyen a la construcción de paz en los territorios y la territorialidad a través de mayores niveles de confianza, tejido social, cohesión social y empoderamiento, entre otros. Por el contrario, la ausencia de un escenario apropiado para actuar en conjunción debilita la posibilidad de potencializar la asociatividad como vehículo de desarrollo rural y del territorio (Unidad Administrativa Especial de Organizaciones Solidarias, 2015). Por tanto, la acción colectiva a través de la asociatividad demuestra que trae beneficios económicos y no económicos que resultan importantes para el desarrollo rural y la construcción de la paz en los territorios (Departamento Nacional de Planeación, 2018).

En efecto, la producción, los rendimientos y el ingreso de los productores tienen un impacto positivo cuya causa es la asociatividad (DNP, 2018). En este sentido, los datos oficiales revelarían que puede existir una correlación positiva entre el Valor Agregado de la producción y el Número de Organizaciones 
en el territorio pertenecientes al sector solidario. La gráfica 1, refleja el Valor Agregado por Departamento (línea continua) y el Número Total de Organizaciones presentes en esos mismos departamentos en el sector rural (línea punteada). Las cifras del Valor Agregado corresponden a la información emitida por el Departamento Administrativo Nacional de Estadística -DANE para el año 2018 a precios corrientes. Por su parte, el número de empresas sin ánimo de lucro con presencia en cada uno de los departamentos hace parte de la información publicada por el Registro Único Empresarial y Social -RUES-.

Gráfico 1. Correlación entre el Valor Agregado y presencia de ESALES por departamento. (Rural -Rural Disperso)

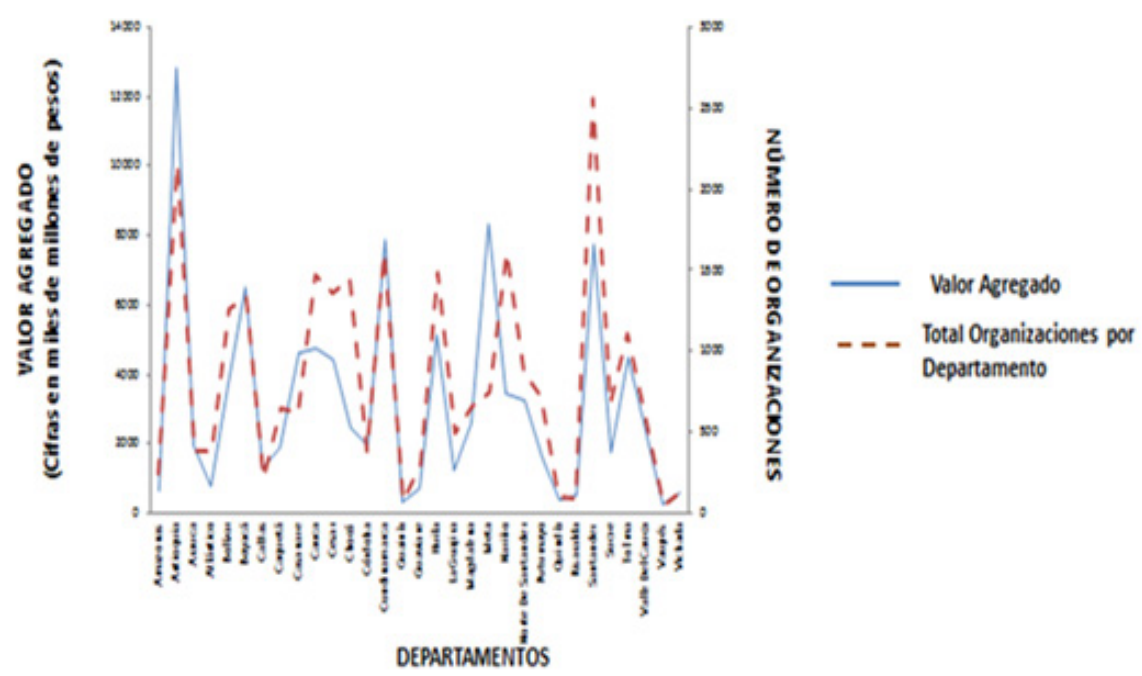

Fuente: Elaboración propia con base en información de DNP, DANE y UAEOS (2014-2018).

De acuerdo con la información anteriormente expuesta en el gráfico, es posible identificar una correlación positiva entre el Valor Agregado (VA) de los departamentos y el número de Organizaciones sin ánimo de lucro en esos territorios. Es decir, habría una probabilidad de que a mayor cantidad de este tipo de organizaciones mayor sería el monto de ese VA. En efecto, al identificar los departamento del Santander como el de Antioquia se observa una relación lineal entre mayor VA en cifras a más organizaciones (en este caso más de 2000 organizaciones reflejan un VA de más de 10.000 millones de pesos). Un componente adicional de análisis relacionado con el VA es el Peso Relativo Municipal en el VA departamental. Este es un indicador que publica el DANE y constituye el aporte porcentual que cada municipio hace al VA del departamento. 
La gráfica 2 expone los diez municipios rurales con mayor peso relativo en su respectivo departamento, que para el caso son Albania y Barrancas (Guajira), Puerto Gaitán (Meta), Tame (Arauca), Leticia (Amazonas), Puerto Inírida (Guainía), San José del Guaviare (Guaviare), Mitú (Vaupés), Puerto Carreño y Cumaribo (Vichada), comparado con el total de organizaciones constituidas en las mismas regiones. En términos generales, se identifica que a mayor sea el número de organizaciones solidarias, mayor es el peso relativo del municipio en el territorio. No obstante, al identificar por territorios los departamentos con mayor VA no necesariamente poseen los territorios con mayor asociatividad. Sin embargo, si se pueden identificar que estos territorios están entre los más golpeados por el conflicto interno y en consecuencia con mayor necesidad de reconfiguración del tejido social y empresarial (Rendón y Gómez, 2020; Correa, 2017; 2018). En este caso desde la economía solidaria para fortalecer el territorio y su desarrollo rural.

Gráfica 2. Los diez municipios con mayor peso relativo y número de organizaciones

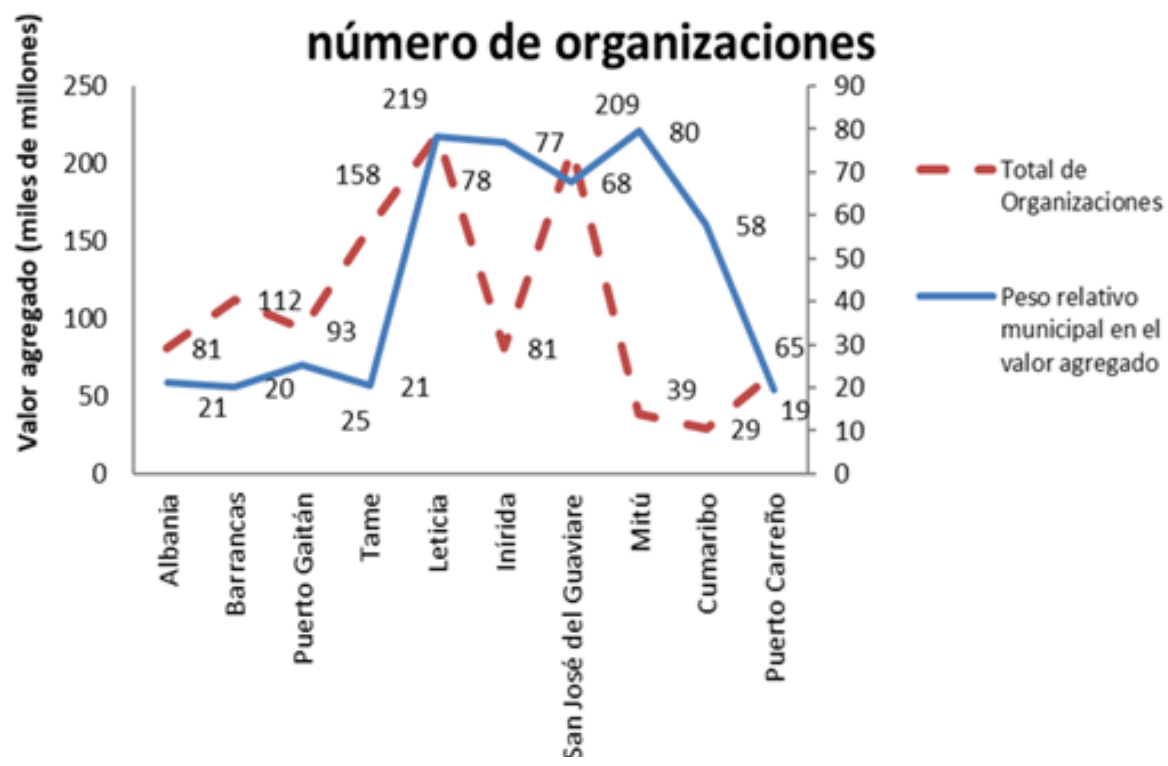

Fuente: Elaboración propia con base en información de la UAEOS y DIAN².

2 Departamento de Impuesto y Aduanas Nacionales-DIAN. 
A su vez, la tabla 1 indica la distribución según tipo de organización por cada uno de los diez municipios con mayor peso relativo en su respectivo departamento teniendo en cuenta la figura jurídica adoptada.

Tabla 1. Los diez municipios con mayor peso relativo en el valor agregado departamental y tipos de organizaciones.

\begin{tabular}{|c|c|c|c|c|c|c|c|c|}
\hline Municlpis & $\begin{array}{l}\text { Pese relaswo } \\
\text { municlpat en } \\
\text { strallet }\end{array}$ & Cooperativas & $\begin{array}{l}\text { Fendos de } \\
\text { Empleades }\end{array}$ & 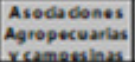 & $\begin{array}{c}\text { Asodadones } \\
\text { Mutuales }\end{array}$ & Coporadones & Fundadones & $\begin{array}{c}\text { Total } \\
\text { Organkadons }\end{array}$ \\
\hline ibsnis & 21 & 15 & 2 & 5 & 0 & 1 & 29 & 73 \\
\hline srrancas & 20 & 6 & 0 & 14 & 0 & 3 & 35 & 78 \\
\hline yerto Gatin & 25 & 11 & 0 & 8 & 1 & 11 & 12 & 68 \\
\hline ame & 21 & 16 & 2 & 65 & 2 & 13 & 23 & 142 \\
\hline eticis & 78 & 24 & 3 & 6 & 14 & 16 & 30 & 171 \\
\hline nirida & 77 & 17 & 2 & 0 & 1 & 11 & 14 & 122 \\
\hline $\begin{array}{l}\text { ian lose del } \\
\text { iudiace }\end{array}$ & $6 B$ & 21 & 2 & 45 & 0 & 31 & 23 & 190 \\
\hline Aitus & 80 & 2 & 0 & 2 & 0 & 7 & 10 & 101 \\
\hline $\begin{array}{l}\text { verto } \\
\text { iarreilo }\end{array}$ & 19 & 3 & 0 & 5 & 0 & 9 & 14 & 50 \\
\hline iumaribo & 58 & 1 & 0 & 1 & 2 & 3 & 3 & 68 \\
\hline
\end{tabular}

Fuente: Elaboración propia con base en información de la UAEOS.

A partir de la firma de los Acuerdos de Paz con las Fuerzas Armadas Revolucionarias de Colombia -FARC- se estableció la implementación de la Reforma Rural Integral -RRI- que contempla, entre otras cosas, la intervención priorizada en 16 zonas que comprenden 170 municipios en 19 departamentos en las áreas más afectadas por el conflicto. El instrumento por medio del cual se logra esa intervención son los Programas de Desarrollo con Enfoque Territorial -PDET ${ }^{3}$ - cuya función es la implementación y gestión de la RRI ${ }^{4}$ (Rúa, et al., 2016).

De acuerdo con la información tomada de la Unidad Administrativa Especial de Organizaciones Solidarias, el Departamento Administrativo Nacional de Estadística y el Registro Único de Economía Solidaria, existiría una correlación positiva entre la presencia de organizaciones solidarias y el VA en los departamentos. Se encuentra que donde hay mayor número de este tipo de organizaciones también hay un alto VA. De ahí que, al observar los diez municipios con mayor peso relativo en el valor agregado departamental, de acuerdo con la figura jurídica adoptada por las organizaciones solidarias, se aprecia que las fundaciones son las que mayor presencia tienen seguidas de las asociaciones agropecuarias y las

3 Instrumento de planificación como de gestión que tiene como objetivo los componentes de la Reforma Rural Integral y las medidas del Acuerdo Final de la Habana en el año 2012.

4 Reforma Rural Integral. 
cooperativas (Barbosa, Merchán y Gómez, 2020; Cueto, Arboleda, Zabala y Echeverry, 2018).

Tabla 2. Organizaciones según forma jurídica en los 129 municipios rurales de las zonas PDET por departamento.

\begin{tabular}{|c|c|c|c|c|c|c|c|c|}
\hline Peparta =ces & Cooprative & 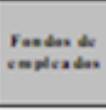 & $\begin{array}{c}\text { Andacines: } \\
\text { oweales }\end{array}$ & 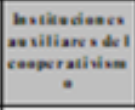 & 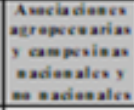 & Carparasiases: & fordadieses: & $\begin{array}{c}\text { Tatal } \\
\text { Ornasizadases }\end{array}$ \\
\hline Inbogra & 83 & 0 & 1 & 0 & 44 & 246 & 14 & 388 \\
\hline Inva & 28 & 2 & 8 & 0 & 168 & 15 & 41 & 262 \\
\hline 3olvar & 67 & 4 & 2 & 0 & 304 & 146 & 59 & 582 \\
\hline zevá & 19 & 0 & 6 & 0 & 247 & 26 & 74 & 372 \\
\hline $20<2$ & 127 & 3 & 2 & 1 & 194 & 27 & 115 & 469 \\
\hline zasar & 71 & 4 & 0 & 0 & 11 & 7 & 92 & 185 \\
\hline $70 \times 6$ & 168 & 11 & 3 & 3 & 0 & 378 & 150 & 713 \\
\hline Córdoba & 12 & 0 & 0 & 0 & 0 & 3 & 7 & 22 \\
\hline haviare & 31 & 2 & 0 & 0 & 61 & 33 & 25 & 152 \\
\hline Ivita & 10 & 0 & 1 & 0 & 19 & 0 & 4 & 34 \\
\hline 2 Couna & 34 & 1 & 0 & 1 & 57 & 4 & 89 & 186 \\
\hline Sera & 15 & 0 & 9 & 0 & 12 & 43 & 20 & 99 \\
\hline iatio & 11 & 2 & 2 & 0 & 105 & 2 & 71. & 193 \\
\hline $\begin{array}{l}\text { Vorte de } \\
\text { Lamandar }\end{array}$ & 47 & 0 & 2 & 0 & 73 & 7 & 21 & 150 \\
\hline venmavo & 49 & 2 & 11 & 0 & 127 & 8 & 65 & 262 \\
\hline ivcre & 33 & 0 & 0 & 0 & 9 & 9 & 41 & 92 \\
\hline Colime & 18 & 0 & 1 & 0 & 48 & 2 & 10 & 79 \\
\hline lotal & $\$ 23$ & 31 & 48 & 5 & 1479 & 956 & $\$ 99$ & 4240 \\
\hline
\end{tabular}

Fuente: Elaboración propia con base en información de la UAEOS.

Lo anterior lleva para tener en cuenta, en el contexto de este documento, la presencia de la economía solidaria en los municipios PDET. En este sentido, se muestra en la tabla 2 la cantidad de organizaciones y formas jurídicas presentes en estas zonas. La información presentada se refiere a los 129 municipios denominados rurales en esas zonas del total de los 170.Ratificando que el grueso de las organizaciones solidarias están en el Choco (713), Cauca (469) y Bolívar con 582. Territorios azotados por la violencia del conflicto armado y en consecuencia departamentos que ejemplifican que la economía solidaria puede ser una estrategia de construcción de la paz como del desarrollo rural de los territorios dado la expansión de organizaciones solidarias y su relación con el VA (González, 2019; Herrera, 2017).

\section{DISCUSIÓN}

En el contexto de cualquier conflicto armado los lazos de confianza y solidaridad, elementos fundamentales de la economía solidaria, tienden a debilitarse (Departamento Nacional de Planeación, 2018). Aun así, y a pesar de que los territorios con un entorno violento pueden disminuir el 
desarrollo económico y el capital social por la circunstancia anteriormente enunciada, las iniciativas colectivas de producción pueden apropiarse de un segmento de las economías de escala y aumentar su poder de negociación (Rendón y Gómez, 2020; Orozco, Forero y Wells, 2013). Por tanto, la conformación y fortalecimiento de organizaciones solidarias con sus distintas formas jurídicas en regiones de posconflicto pueden aumentar la cohesión social necesaria para la reconstrucción de dichos territorios afectados por la violencia. (Unidad Administrativa Especial de Organizaciones Solidarias, 2016). En contraposición, la participación en organizaciones productivas disminuye en aquellos lugares con alta presencia de grupos armados al margen de la ley (Gómez, Barbosa, y Rojas, 2021; Gáfaro, Ibáñez y Justino, 2014). En efecto, el presente ejercicio investigativo se sintoniza con lo antes expuesto al reflejar que donde hay mayor VA por departamento existe también una mayor concentración de Organizaciones Solidarias. Ratificando que donde se genera a asociatividad existe posibilidades de mejorar la calidad de vida de las personas, en virtud que existen relaciones de cooperación, de empatía que ayudan en resacar el tejido social desconfigurado por 60 años de conflicto armado interno (Rodríguez, Laverde y Barbosa, 2021; Osorio, 2017; Pepesca, 2015; Meira, 2014).

Colombia se han presentado desarrollos teórico-conceptuales gracias a experiencias diversas y existe un marco normativo de la Economía Solidaria presente en la Ley 454 de 1998. El marco normativo colombiano busca establecer una manera diversa y plural de identificar las expresiones de Economía Solidaria como identidad basada en una lógica económica con relaciones de solidaridad, reciprocidad y cooperación para ampliar la visión empresarial. La comunidad establece factores económicos donde el trabajo autogestionado condensa factores que se afianzan en las empresas de economía solidaria (Gómez, 2020; En efecto el ambiente jurídico que presenta el Estado colombiano referente a la economía solidaria va en relación en la dinamización de lo rural y lo urbano tal como se presentó en el artículo en cabeza del rector de la economía solidaria en Colombia como la Unidad Administrativa Especial de organizaciones solidarias (UAEOS, 2019; 2016)

\section{CONCLUSIONES}

El presente artículo tuvo como objetivo general describir la economía solidaria como posible dinamizadora del desarrollo rural y de la construcción de la paz en Colombia en el periodo 2014-2018. Para ello, se utilizaron la metodología cualitativa y cuantitativa por medio de los datos de instituciones colombianas reconocidas. A modo exploratorio se relacionaron variables como lo fueron el Valor Agregado y las Organizaciones Solidarias. Los 
resultados sugieren que a mayor número de organizaciones, establecen la hipótesis de que a mayor número de organizaciones mayor es el Valor Agregado del territorio. Sin embargo, para poder afirmar esta hipótesis es necesario efectuar un análisis econométrico para poder identificar las variables exógenas y endógenas como realizar la medición. No obstante, este ejercicio está por fuera del artículo y abre la puerta para construir un nuevo artículo

La Economía Social representa una mirada diferente a la ortodoxia económica donde la cooperación, la solidaridad y la ayuda mutua se erigen como aspectos fundamentales en las relaciones entre comunidades con especial énfasis en el desarrollo integral del ser humano. Precisamente, en tiempos de construcción de paz gracias al acuerdo firmado entre el gobierno de Colombia y el grupo armado FARC-EP, la posibilidad de un desarrollo rural se posibilita gracias al encuentro de actores con fines en común y donde el cooperativismo, el comercio justo y el trabajo digno constituyen conceptos relevantes

Las organizaciones solidarias contribuyen en mejorar a las comunidades y su entorno, facilitando además las oportunidades de cumplir sueños que van más allá de necesidades básicas insatisfechas, puesto que generando o mejorando sus emprendimientos asociativos, y con un uso de los excedentes basados en principios solidarios, se logra una mejor distribución de la riqueza, un destino diferente para los jóvenes que aún siguen en el campo y sostenibilidad ambiental en las regiones. En virtud que el mejoramiento de calidad de vida, tanto de las personas vinculadas a dichas organizaciones, como de las zonas en las que se localizan. En particular, en escenarios afectados por la violencia, la asociatividad puede convertirse en un mecanismo para acceder a los mercados y aumentar el poder de negociación de los productores a través de la cohesión social cuya consecuencia, entre otras, es el desarrollo rural.

\section{BIBLIOGRAFÍA}

Álvarez, J. y López, M. (Comps.). (2018). Apuntes para el fomento de la asociatividad solidaria y el logro de los objetivos de desarrollo sostenible. Unidad Administrativa Especial de Organizaciones Solidarias (UAEOS); Centro de Investigación, Documentación e Información de la Economía Social, Pública y Cooperativa (CIRIEC).

Álvarez, J. (2016). Articulación de políticas públicas dirigidas a las cooperativas. Propuestas para el caso colombiano en el marco de la convivencia pacífica y la internacionalización. Lisboa: Instituto Superior de Ciências Sociais e Políticas da Universidade de Lisboa. Tese elaborada para obtenção do grau de Doutor em Ciências Sociais 
Amaya, A. L. (2013). Las fallas de mercado, su vinculación con los procesos de generación y transferencia de conocimiento en espacios rurales. Revista Electrónica de Investigación en Ciencias Económicas, 1(2), 134 166.

Amaro, R. (2009). A Economia Solidária da Macaronésia - Um novo conceito. Revista Economia Solidária (1), 11-30.

Barbosa, E., Vargas, H., y Gómez, D. (2020). Breve estudio bibliométrico sobre economía solidaria. Revista Cooperativismo y Desarrollo Instituto de Economía Social y Cooperativismo. 28(118),80-94

Barbosa, E., Merchán, O., y Gómez, D. (2020). La economía solidaria como potenciador del desarrollo rural y de la construcción de la paz en Colombia. Ponencia. IV Seminario Internacional de Desarrollo y Territorio, Universidad de la Salle, Bogotá, Colombia.

Barbosa, E., y Gómez, D. (2020). Macro proyecto: Análisis, desarrollo, fomento y gestión del talento humano en el municipio de Aracataca, Magdalena desde la economía solidaria (2020-2022). Micro proyecto I: Analizar la categoría economía social y solidaridad (2020) Micro proyecto II: Fomento y fortalecimiento de circuitos cortos de comercialización en el territorio de Aracataca, Magdalena (2021). Ponencia. RITA: Red de Investigaciones de Tecnología Avanzada. Universidad Distrital.

Barbosa, E., y Gómez, D. (2021).Economía solidaria y construcción de paz. Revista Sur. Obtenido en : https://www.sur.org.co/economia-solidaria-yconstruccion-de-paz/

Barbosa, E., Insuasti, E., y Gómez, D. (2020). Las compras públicas y los circuitos cortos de comercialización como estrategias para la reactivación económica de las zonas rurales afectadas por COVID 19. Ponencia. III Congreso CATATUMBARİ. Universidad Francisco de Paula Santander -Ocaña. Colombia.

Bértola, L., y Ocampo, J. (2012). El desarrollo económico de América latina desde la independencia. México D.F.: Editorial Fondo de Cultura Económica.

Berzosa, C. (1999). Mercado, estado y economía mundial. Revista de Economía Mundial, 29-50.

Caillé, A. (2009), Sobre los conceptos de economía en general y de economía solidaria en particular. En ¿Qué es lo económico? Materiales para un debate necesario contra el fatalismo. Buenos Aires, Argentina: Ediciones CICCUS.

Cárdenas. D., y Vallejo, C. (2016). Agricultura y desarrollo rural en Colombia 2011-2013: una aproximación. Apuntes de Cenes.35:(62),87-123

Ceña, F. (1993). El desarrollo rural en sentido amplio. En R. Ramos, E. y P. Caldente y del Pozo. El desarrollo rural andaluz a las puertas del siglo XXI. Congresos y Jornadas 32/93. Andalucía, España: Dirección General 
de Investigación, Tecnología y Formación Agroalimentaria y Pesquera, Consejería de Agricultura y Pesca, Junta de Andalucía.

Chávez, R. (2012). Las políticas públicas y las cooperativas. Ekonomiaz Revista Vasca de Economía, 169-200.

Chaves, R. (2006). La Economía Social en la Unión Europea. Comité Económico y Social Europeo.

Coraggio, J. (2011). Economía Social y Solidaria. El trabajo antes que el capital. (1 a ed.). A. Acosta, y E. Martínez, (Edits.). Quito, Ecuador: Abya Yala. Universidad Politécnica Salesiana.

Correa, G. (2018). Agrociencias y cambio climático. Ediciones Unisalle. Bogotá: Colombia.

Correa, G. (2017). Agrociencias y territorio. Ediciones Unisalle. Bogotá: Colombia.

Cruz, V. (2007). Territórios, identidades e lutas sociais na Amazônia. En: Araujo, F. G. B.; Haesbaert, R. Identidades e territórios: questões e olhares contemporâneos (pp. 93 - 122). Rio de Janeiro, Brasil: Access Editora.

Cueto, E., Arboleda, O., Zabala., y Echeverry, F. (2018). Una década de economía social y solidaria en Colombia: análisis de la producción investigativa y académica 2005 -2015.Bogotá, Colombia: Corporación Universitaria Minuto de Dios - UNIMINUTO y Universidad Católica Luis Amigó.

DANE, (2018). Boletín Técnico de Colombia. Pobreza monetaria en Colombia. DANE. Obtenido en: https://www.dane.gov.co/files/ investigaciones/condiciones_vida/pobreza/2018/bt_pobreza_ monetaria_18.pdf

Delgado, J., y Pardo, J. (2014). Valor agregado municipal, un ejercicio de categorización empleando componentes principales y análisis de conglomerados. Revista IB.4:(81)150-164.

DNP. (2018). Departamento Nacional de Planeación. Asociatividad, desarrollo agrario integral y construcción de paz: La acción colectiva como estrategia para aumentar el acceso a recursos y mercados en zonas rurales. Bogotá, Colombia: DNP.

Estivill, J. (2012). Espacios públicos y privados. Construyendo diálogos en torno a la Economía Solidaria. Revista crítica de ciencias sociales. (84), 101-113.

Echeverri, J. (2004). Territorio como cuerpo y territorio como naturaleza: ¿diálogo intercultural? In: Surrallés, A.; García, P. Tierra adentro: territorio indígena percepción del entorno. (pp. 259 -276). Lima, Perú: Iwgia.

Espinosa, O. (2009). ¿Salvajes opuestos al progreso?: aproximaciones históricas y antropológicas a las movilizaciones indígenas en la Amazonía peruana. Revista Antropológica, (27), 123 - 168. 
Gáfaro, M., Ibáñez, A. M., y Justino, P. (2014). Collective Action and Armed Group.

Gómez, D., Aldana, K., y Rodríguez, R. (2021). Antropologías del desarrollo, enfoques alternativos y postdesarrollo. Breve revisión de conceptos y apuntes críticos. Población y Desarrollo. 27 (52): 108 - 122.

Gómez, D., Barbosa, E., y Rojas, J. (2021). Prueba piloto del Plan Nacional de Fomento de la Economía Solidaria y Cooperativa Rural - PLANFES. Estudio del caso del municipio de San Antero, Córdoba, Colombia. Otra economía: revista latinoamericana de economía social y solidaria. 14: (25), 77-94.

Gómez, D. (2020). Criaturas en el encierro: reflexiones en tiempos de coronavirus. En J. Wilches, Proteodante, \& A, Solidaridad en época de pandemia. Aracataca, Magdalena: cuna del premio Nobel de literatura (págs. 65-77). Bogotá: Editorial Universidad Distrital de Colombia.

Gómez, D. Carranza, Y., Ramos, C. A. (2017a). Ecoturismo, turismo experiencial y cultural. Una reflexión con estudiantes de Administración de Turismo. Revista TEMAS, 3:(11), 229 - 236.

Gómez, D. Carranza, Y., y Ramos, C. A. (2017b). Aportes de negociadores internacionales para el crecimiento, la innovación, el emprendimiento y la competitividad de las empresas colombianas. Revista FACCEA 7:(2), 157 - 164.

Gómez, D., Carranza, Y., y Ramos, C. (2016). Revisión documental, una herramienta para el mejoramiento de las competencias de lectura y escritura en estudiantes universitarios. Chakiñan, Revista de Ciencias Sociales y Humanidades. (1), 46-56.

Gómez, D., y Rojas, W. (2017). Política agraria, postconflicto e inclusión. En el libro Tomo II de Ágora Latinoamericana Construcción de Espacios Regionales: Inclusión Social Latinoamericana. Universidad de Ciencias Aplicadas y Ambientales U.D.C.A Asociación de Universidades de América Latina y el Caribe para la Integración AUALCPI.

Gómez, D. (2015). Introducción al Neoliberalismo. En: Neoliberalismo y Educación. Ed Gómez D. Bogotá, Colombia: Editorial CUN.

Gonzáles, A. (2019). Gobernabilidad y educación rural en el marco del conflicto armado en Colombia. Tesina de Maestría en estudio y Gestión del desarrollo. Universidad de la Salle. Obtenido en: https://ciencia. lasalle.edu.co/cgi/viewcontent.cgi?article=1572\&context=lic_lenguas

Gottman, J. (1973). The significance of territory, Charlottesville, United States of America: The University Press of Virginia.

Guerra, P. (2014). Socioeconomía de la solidaridad. Una teoría para dar cuenta de las experiencias sociales y económicas alternativas ( 2 a ed.). Bogotá, Colombia: Ediciones Universidad Cooperativa de Colombia.

Kalmanovitz, S. (2019). Nueva historia económica de Colombia. Edición revisada y actualizada. Barcelona: España: Taurus. 
Hernández, C., Fernández, P., y Baptista, A. (2006). Metodología de la investigación, México D. F.: México: Mc Graw Hill.

Herrera, A. (2017). Las políticas públicas como alternativa estructural para el desarrollo rural solidario y el posconflicto colombiano. Revista de la Universidad de La Salle, (74), 71-100.

Laville, J. (2004). El marco conceptual de la Economía Solidaria, en Laville, Jean Louis (Ed.) Economía Social y Solidaria. Una visión europea, Buenos Aires: Altamira, pp. 207-236.

Laville, J., Levesque, B., y Mendell, M. (2006). The Social Economy. Diverse approaches and practices in Europe and Canada. Quebec, Canada: Bibliothéque et Archives Canada.

Laville, J. (2007). L' economie Solidaire, une perspective internationale. C. H. Sociologie Ed. Martorell, M., y Santos, J. (2012). Manual de historia política y social de España (1808-2011). Barcelona, España: rba.

Laverde, M., Almanza C., Gómez, D., y Serrano , C. (2020). El Capital Relacional como Recurso Diferencial y Valioso para las Empresas. PODIUM, (37), 57-70. https://doi.org/10.31095/podium.2020.37.5

Losada, J., Valencia, S., y Gómez, D. (2018). La gestión de talento humano en el desarrollo organizacional. CrearE, Cuadernos de Emprendimiento,3,62-69.

Ley 454 de 1998. Función Pública. Gestor Normativo. Obtenido en: https:// www.funcionpublica.gov.co/eva/gestornormativo/norma.php?i=3433

Machado, A. (2005). Las políticas y el modelo de desarrollo agropecuario. En: Absalón Machado (coord.). La academia y el sector rural 5. Bogotá, Colombia: Universidad Nacional-Facultad de Ciencias Económicas-CID.

Medeiros, R. (2009). Território, espaço de identidade. En: Saquet, M.A.; Sposito, E. S.Territórios e territorialidades: teorias, processos e conflitos. (pp. 217 - 227). São Paulo, Brasil: Expressão Popular.

Maldonado, E. (2016). Complejidad de las ciencias sociales. Y de otras ciencias disciplinas. Ciencia y Sociedad. Bogotá, Colombia: Ediciones desde abajo.

Maldonado, E. (2007). Complejidad: ciencia, pensamiento y aplicación. Bogotá, Colombia: Universidad Externado de Colombia.

Molina, J., Aranda, Y., y Lesmes., A. (2018). Desafíos para la implementación de políticas de desarrollo rural con enfoque territorial en Colombia. Editorial Universidad Nacional de Colombia.

Manzanal, M. (2006). Regiones, Territorios e Institucionalidad del Desarrollo Rural. En M. Manzanal, G. Neiman, Y., y M. Lattuada, Desarrollo Rural Organizaciones, Instituciones y Territorio (págs. 21-50). Buenos Aires, Argentina: CICCUS.

Martín, J. (1983). El significado de las externalidades. Cuadernos de Economía, 405-434. 
Meira, F. (2014). Liminal organization: Organizational emergence within solidary economy in Brazil. Organization $21:$ (5) 713-729.

Oxfam (2017). Radiografía de la desigualdad: lo que nos dice el último censo agropecuario sobre la distribución de la tierra en Colombia.Oxfam.

Orozco, L. E., Forero, C., y Wills, E. (2013). Inseguridad rural y asociatividad. Bogotá, Colombia: Uniandes.

Osorio, M. (2017). Modos de vida vivibles: Economía(s) Solidaria(s) y sostenibilidad de la vida. Barcelona, España: Universidad Autónoma de Barcelona.

Pérez, G. (2018). Territorio Amazónico peruano: un debate teórico sobre los conceptos de territorio, des - re-territorialización y colonialidad. Revista Huellas, 22:(1), Instituto de Geografía, EdUNLPam: Santa Rosa. Obtenido en: http://cerac. unlpam.edu.ar/index.php/huellas

Pérez, N., y Uribe, V. (2016). Reflexiones para conceptualizar territorio solidario. Revista ÁGORA.usb, 16 (2), 533- 546.

Pérez, E y Pérez. (2002). ¡El sector rural en Colombia y su crisis actual! Cuadernos de Desarrollo Rural (48),35-58.

Pérez, E. (1998). Una visión del desarrollo rural en Colombia. Cuadernos de Desarrollo Rural, (41).

Popescu, D. (2015). Solidary and Social Market Economy. Present Interest in Leon Bourgeois and Charles Gide. doi:10.1016/S2212-5671(15)003056.

Raffestin, C. (1980) Por una geografía del poder. Traducción y notas Y. Villagómez Velázquez (2011). Obtenido en: http://elitesydesarrollorural. pieb. org/files/b02_claude_raffestin. Pdf

Razeto, L. (1992). Fundamentos de la teoría económica comprensiva. Santiago de Chile, Chile: Ed. pet

Rendón J., y Gómez, D. (2020). Circuitos cortos y verdes. Alimentos si hay. Revista sur. Obtenido en: https://www.sur.org.co/circuitos-cortos-yverdes-alimentos-si-hay/

Rendón, J. (2007). TLC y exportaciones: la apuesta por la estabilidad del comercio. Revista Equidad \& Desarrollo.25-37.

Rendón, J., Nieto, P., y Ángel, D. (2005). En la búsqueda de enfoques para el desarrollo. A propósito del debate entre el Consenso de Washington y el Fórum de Barcelona. Revista Equidad \& Desarrollo. (4),47-64.

Restrepo, J., y Bernal, A. (2014). La cuestión agraria. Tierra y posconflicto en Colombia. Bogotá: Penguin Random House.

Rodríguez, D., Laverde, M., y Pérez, E. (2021). Short Commercialization Circuits in Local Supply Chains: Economic Revival in the Pandemic. South Asian Journal of Social Studies and Economics, 12(2), 1-10. https://doi. org/10.9734/sajsse/2021/v12i230299. 
Rúa, S., Monroy, V., Peñuela, J., Pérez, P., Calderón, A., Arenas, C., y Jiménez, H. (2016). Integración económica solidaridad en territorio. Bogotá: UAEOS y Universidad Cooperativa.

Rubio, G., Téllez, C., y Gómez, D. (2019). Análisis de los factores que componen un sistema de gestión empresarial: estudio de caso para la Dirección de Vivienda. Revista Nacional de Administración.10:(2)47-60

Sánchez, A., y Herrera, E. (2017). Desarrollo Rural Local: Un enfoque de desarrollo para el reconocimiento de la diversidad rural y los actores locales. Tesis de maestría. Obtenido en: http://repository. lasalle.edu.co/bitstream/handle/10185/21359/72121216_2017. pdf?sequence=1\&isAllowed=y

Silva, A. (2017). Factores que explican el éxito de organizaciones de economía solidaria constituidas durante el proceso de los diálogos de Paz en Colombia: hallazgos de emprendimiento en el sector cooperativo. España. Universitat de València. Tesis elaborada para obtener el título de doctora en Dirección de Empresas.

Stiglitz, J. (2020). Capitalismo progresista. La respuesta a la era del malestar. Taurus, Barcelona: España.

Stiglitz, J. (2012). El precio de la desigualdad. Madrid, España: Taurus.

Stiglitz, J. (2010). Regulación y fallas. Revista Institucional, 12-23.

Trivelli, C., Escobal, J. y Revesz, B. (2009). Desarrollo rural en la Sierra. Aportes para el debate. IEP. CIPCA. GRADE. CIES. Obtenido en: http:// www.grade. org.pe/download/pubs/libros./Desarrollo.pdf

Unidad Administrativa Especial de Organizaciones Solidarias - UAEOS. (2019). La economía solidaria en el Plan Nacional de Desarrollo "Pacto por Colombia, Pacto por la Equidad" 2018-2022. Ley 1955 de 2019.

Unidad Administrativa Especial de Organizaciones Solidarias y Centro de Investigación, UAEOS Y Documentación e Información de la Economía Social, Púbica y Cooperativa. (2018). Apuntes para el fomento de la asociatividad solidaria y el logro de los objetivos de desarrollo sostenible. Bogotá: UAEOS Y CIRIEC.

UAEOS. (2016). Unidad Administrativa Especial de Organizaciones Solidarias. Integración económica solidaria en territorio. Bogotá, Colombia: UAEOS.

UAEOS. (2015). Unidad Administrativa Especial de Organizaciones Solidarias Marco para el fomento de la economía solidaria en territorios rurales en Colombia. Bogotá, Colombia: UAEOS.

Useche, O. (2008). Los nuevos sentidos del desarrollo. Ciudadanías emergentes, paz y reconstrucción. Bogotá, Colombia: Universidad Minuto de Dios.

UNESCO (2010). Organización de las Naciones Unidas para la Educación, la Ciencia y la Cultura. Llegar a los marginados. Informe sobre la Educación 
para Todos en el Mundo 2009. Paris, Francia. UNESCO. Obtenido en: http://www.unesco.org/es/efareport/reports/2010-marginalization/

Vargas, A., y Sánchez, C. (2020). Análisis de evolución de la asistencia técnica y el fomento de cooperativas rurales en Colombia. Cooperativismo \& Desarrollo, 27(116)1-22. doi: https://doi.org/10.16925/23824220.2020.01.03

Vásquez, L., y Jiménez, J. (2012). Economía solidaria: patrimonio cultural de los pueblos. No. 306.

Vergara, W. (2011). Desarrollo del subdesarrollo o nueva ruralidad para Colombia. Cartografías del desarrollo rural. Revista de la Universidad de la Salle, (55),33-66. 